\title{
Anthocyanin Pigments: Comparison of Extract Stability
}

\author{
ALAN BAUBLIS, ART SPOMER, and M.D. BERBER-JIMÉNEZ
}

\begin{abstract}
Stabilitics of anthocyanins from Concord grapes, rcd cabbage, tradescantia and ajuga were studied. RP-HPLC analysis was used to characterize individual anthocyanins and spectrophotometric analysis for all pigments. Anthocyanins from tradescantia were much more stable than the other extracts. Such increased stability was attributed to its intrinsic nature.
\end{abstract}

Key Words: Anthocyanins, stability, Concord grapes, cabbage, tradescantia, ajuga, extract stability

\section{INTRODUCTION}

THERE IS INCREASING USE of natural food colorants (Francis, 1992) and anthocyanins provide color for products such as red wines, jams and fruit juices. Anthocyanins as colorants commercially have been limited because of their lack of stability and difficult purification. Commercially successful food colorants employing anthocyanins usually contain pigments with relatively simple structures (Shi and Francis, 1992). Anthocyanins from grapes include mono- and diglucosides of five different aglycones with the addition of monoacylation. The realization that acylation of the molecule led to increased stability led to commercial development as food colorants of the diacylated triglucosides of cyanidin found in red cabbage (Labell, 1990).

Anthocyanins derived from ornamental plants have in general shown a higher degree of acylation than common fruit and vegetable sources. Davies and Mazza (1992) found that the main anthocyanin from Monarda fistulosa was pelargonidin 3,5-diglucoside acylated with $p$-coumaric and malonic acid (Davies and Mazza, 1992). Other sources of highly acylated anthocyanins include Lobelia erinus, Tradescantia pallida, and Zebrina pendula. The acylated anthocyanins have been of interest because of the acyl group substitution relation to stability (Teh and Francis, 1988). Anthocyanins from tradescantia, a plant related to Zebrina, was reported by Shi et al. (1993) to have excellent stability when compared to a commercial grape extract and blackberry extract (Shi et al., 1992). The anthocyanins of Ajuga reptans cultures was suggested by Callebaut et al. (1990) as useful food colorants, due to their high acylation.

Our objective was to monitor the stability of structurally different anthocyanins under controlled conditions.

\section{MATERIALS \& METHODS}

RED CABBAGE DRIED EXTRACT was obtained from San-Ei Chemical Industries, Ltd. (Osaka, Japan) and used without further preparation. Leaves of tradescantia (Tradescantia pallida, v. 'purple heart') were collected from greenhouse-grown plants (Department of Horticulture, University of Illinois, Urbana, IL) and freeze dried immediately using a Vacudyne Freeze Drier (Chicago, IL) chamber equipped with a Copeland pump (Sidney, $\mathrm{OH}$ ). Concord grapes were obtained from Michigan (Stresling Winery) and freeze dried, in the same manner as the tradescantia leaves, within $24 \mathrm{hr}$. Ajuga leaves were collected in Urbana and extracted immediately.

Authors Baublis and Berber-Jimenez are with the Dept. of Food Science and Author Spomer is with the Dept. of Horticulture, Univ. of Illinois, 1208 W. Pennsylvania Ave., Urbana, IL 61801.

\section{Extraction of anthocyanins}

Samplcs werc extracted at room temperature $\left(\sim 23^{\circ} \mathrm{C}\right)$ overnight in an extraction medium consisting of reagent grade $3 \%$ trifluoroacetic acid (TFA) in water $(v / v)$. Extracts were filtered under vacuum through Whatman No. 1 filter paper, and then loaded on an Amberlite XAD-7 resin column (Aldrich Chemical Company, Inc., Milwaukee, WI) washed with $300 \mathrm{~mL}$ of distilled water and eluted with acetonitrile. Extracts were then concentrated to dryness with a Büchi rotary evaporator (Switzerland) connected to a Welch high vacuum pump (Skokie, IL) at $30^{\circ} \mathrm{C}$. After evaporation, dried samples were placed in a desiccator over calcium sulfate to remove any remaining water. The resulting dried pigments were then used for stability studies.

\section{Preparation of samples for stability study}

Extracts were dissolved in $5 \mathrm{~mL}$ of sodium citrate buffer solution at pH 3.5, then transferred to screw-top test tubes with an Eppendorf pipette (Westbury, NY) to give $1 \mathrm{~mL}$ of extract per tube. The tubes were degassed with helium, sealed with Teflon tape and capped immediately to eliminate oxygen transfer into the tube.

\section{Light exposure chamber}

The light exposure chamber consisted of a water bath surrounded by fluorescent lamps. It was constructed from a $25 \mathrm{~cm}$ long piece of 30.4 $\mathrm{cm}$ diameter clear acrylic cylinder $(0.4 \mathrm{~cm}$ wall) sealed to a flat acrylic base $(0.4 \mathrm{~mm}$ thick). The specimen tubes were supported vertically around the inside wall of the bath at a distance of about $3 \mathrm{~cm}$ from the lamps (including $0.5 \mathrm{~cm}$ water and $0.4 \mathrm{~cm}$ acrylic). Bath temperature was maintained at $30^{\circ} \pm 1^{\circ} \mathrm{C}$ with a Haake D1 (Germany) circulating temperature controller immersed in the center of the bath. An irradiance of $250 \mu \mathrm{mole} \mathrm{sec}^{-1} \mathrm{~m}^{-2}$ (measured with a Li-Cor LI-190SB Quantum Sensor, Lincoln, NE) was provided at the sample location by eight circular $40 \mathrm{~W}$ cool white fluorescent lamps (Sylvania FC16T9/CW/RS) stacked around the bath.

\section{Visible spectroscopy}

Dried extract of known weight was dissolved in sodium citrate buffer solution and then diluted with $0.1 \mathrm{~N} \mathrm{HCl}$. Volume was adjusted for each extract to give an absorbance reading between 0.4-0.6 units (Wrolstad, 1976). The absorbance of the samples was read at $525 \mathrm{~nm}$ using a Beckman DU-50 spectrophotometer (Irvine, CA) connected to an Epson RX80 printer (Japan). Total anthocyanin concentration was calculated based on cyanidin 3-glucoside molar absorptivity $26,900 \mathrm{~L} \mathrm{~mol}^{-1} \mathrm{~cm}^{-1}$ (Jurd and Ansen, 1966), and the molecular weight corresponding to the major anthocyanin in each respective extract.

Visible spectra of samples at various $\mathrm{pHs}$ were measured with the same instrument. Samples were dissolved in appropriate buffer solution and allowed to equilibrate $1 \mathrm{hr}$ before measurements. Sample cells of 1 $\mathrm{cm}$ length were used, and all measurements were at ambient temperature $\left(\sim 23^{\circ} \mathrm{C}\right)$.

\section{High performance liquid chromatography}

The equipment for HPLC consisted of a Hitachi L-6200A Intelligent Pump (Tokyo, Japan), a Hitachi L-4500 Diode Array Detector (Tokyo, Japan) and a Rheodyne (Cotati, CA) 7125 injector valve with a $500 \mu \mathrm{L}$ loop. A YMC S5 120A ODS-AM guard column connected to a YMCPack ODS-AM $(250 \times 4.6 \mathrm{~mm}), 5 \mu$ particle size, column (Wilmington, NC) was used for pigment separation. Data were collected and processed with Hitachi Model D-6500 Chromatography Data Station Software (Tokyo, Japan) loaded on a Gateway $2000486 \mathrm{DX} 2$ personal computer (N. Sioux City, SD). 

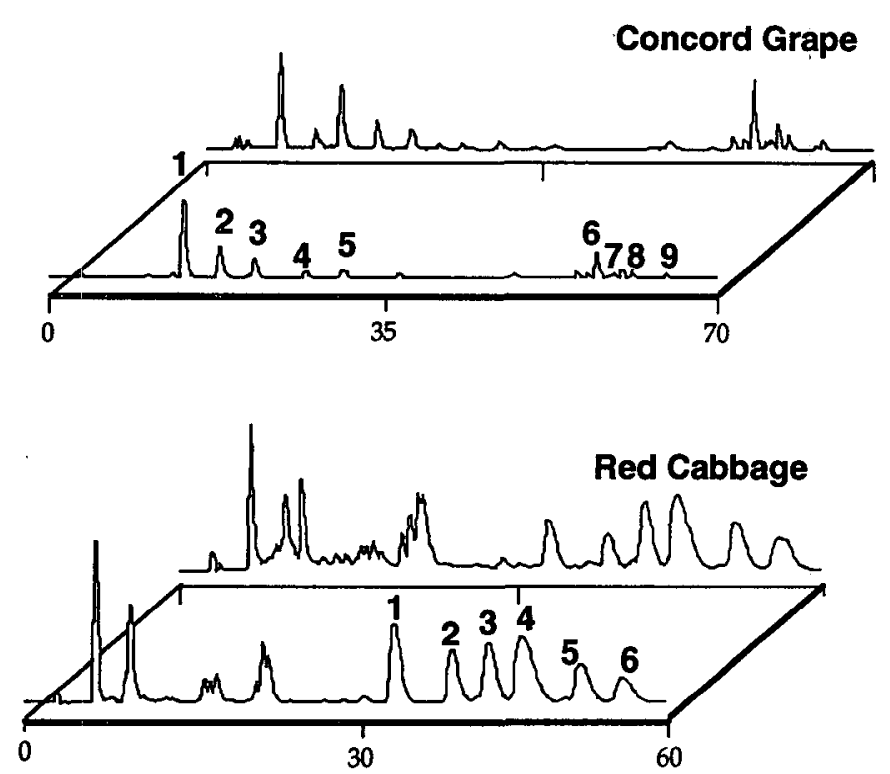

Fig. 1-HPLC chromatograms monitored at $\lambda=520 \mathrm{~nm}$ (front) and $\boldsymbol{\lambda}=300 \mathrm{~nm}$ (back). Concord grape peaks 1-5 are 3-glucosides of delphinidin, cyanidin, petunidin, peonidin, malvidin and peaks 6-10 are 3-glucoside-p-coumarates of delphinidin, cyanidin, petunidin, malvidin. Red cabbage peaks $1-3$ are cyanidin 3-glucosides acylated with one mole of sinapic, ferulic and $p$-coumaric acids respectively; 4-6 are cyanidin 3-sambubioses with one mole of sinapic and one of sinapic, ferulic and $p$-coumaric acids respectively. See Fig. 3 for complete structures of red cabbage peaks.

Solvent systems and elution profiles were optimized to separate anthocyanins for each individual extract. All separations were performed at room temperature $\left(\approx 20-25^{\circ} \mathrm{C}\right)$. All solvents were of HPLC grade with exception of tetrahydrofuran (THF) which was reagent grade. All solvents were pre-filtered through a $0.45 \mu$ nylon filter and stirred under vacuum to degas them. Solvent $A$ was $10 \%$ formic acid in water (v/v). Separation of tradescantia anthocyanins was performed using an isocratic elution of $15 \%$ methanol:THF (1:1) at a flow rate of $0.9 \mathrm{~mL} \mathrm{~min}^{-1}$. Ajuga anthocyanins were separated with an isocratic elution of $9 \%$ methanol:THF (1:1) at a flow rate of $1 \mathrm{~mL} \mathrm{~min}{ }^{-1}$. Red cabbage anthocyanins were separated using methanol:THF (1:1) as solvent $B$, the solvent system consisted of 5-10\% B in $15 \mathrm{~min}$ followed by $10 \%$ B isocratic for $45 \mathrm{~min}$, at a flow rate of $1 \mathrm{~mL} \mathrm{~min}{ }^{-1}$. Separation of Concord grape anthocyanins required a gradient system with acetonitrile as solvent $B$. The elution profile was: $0-5 \mathrm{~min}, 5-8 \%$ B (linear); $5-20 \mathrm{~min}, 8-11 \%$ B; $20-50 \mathrm{~min}, 11-14 \% \mathrm{~B} ; 50-70 \mathrm{~min}, 20-30 \% \mathrm{~B}$ the flow rate was 1 $\mathrm{mL} \min ^{-1}$. Peak area for analysis was calculated using the Hitachi Model D-6500 Chromatography Data Station Software.

Semi-preparative HPLC was performed on the red cabbage extract to collect individual pigments of interest. Semi-preparative HPLC was performed using a YMC-Guardpack ODS-AM $(30 \times 10 \mathrm{~mm})$ guard column connected to a YMC-Pack ODS-AM $(250 \times 10 \mathrm{~mm})$ column (Wilmington, NC). Elution profile was the same as stated earlier with exception of flow rate and injection loop size. For semi-preparative HPLC flow rate was increased to $5.0 \mathrm{~mL} \mathrm{~min}-1$ and an injection loop of 1.0 $\mathrm{mL}$ was used. Also a detector fiow cell of $0.5 \mathrm{~mm}$ width was used for semi-preparative HPLC. Individual peaks were collected manually and concentrated to dryness under vacuum before analyzing by mass spectrometry (MS)

Low resolution MS was done using fast atom bombardment (FAB) MS. The instrument was a FISONS VG analytical ZAB-SE using a dithiothreitol/dithioerythrotol (3:1) matrix.

\section{RESULTS \& DISCUSSION}

HPLC CHROMATOGRAMS of the four extracts (Fig. 1 and 2) monitored at $520 \mathrm{~nm}$ (front) show pigments only, while those monitored at $300 \mathrm{~nm}$ (back) show pigments and other coextracted compounds. Concord grape and red cabbage extracts showed 20 and 15 different anthocyanins respectively. The peak assignment was based on previously reported data (Lea, 1988;
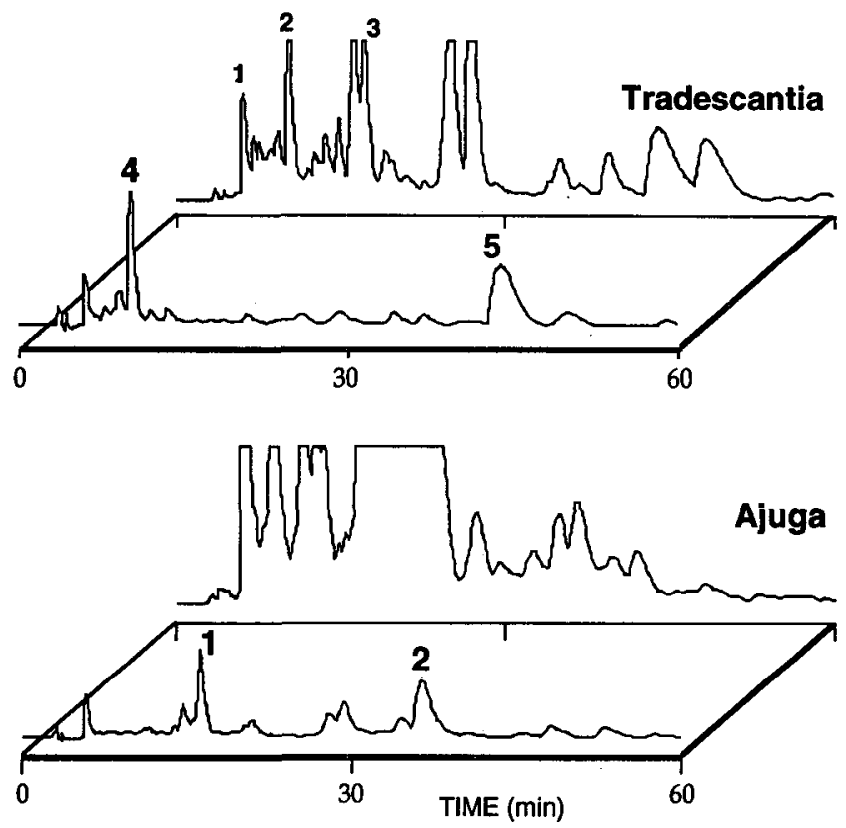

Fig. 2-HPLC chromatograms monitored at $\lambda=520 \mathrm{~nm}$ (front) and $\lambda=300 \mathrm{~nm}$ (back). Tradescantia peaks are 1: chlorogenic acid, 2: caffeic acid, 3: rutin, and 4 and 5: minor and major unidentified anthocyanins. Peak 1 and 2 on ajuga are the minor and major unidentified anthocyanins.

Mazza and Miniati., 1993) and MS data for the cabbage pigments (Fig. 3). Extracts from both red cabbage and Concord grape contained few UV absorbing compounds aside from the anthocyanins. This indicated that stability of these extracts was not improved by intermolecular copigmentation effects.

Tradescantia's and ajuga's major anthocyanins (Fig. 2) have tentatively been assigned as cyanidin derivatives glycosylated and acylated in various positions. The major anthocyanin of ajuga was identified as a glucosylated cyanidin, acylated with p-hydroxycinnamic acid, ferulic acid and malonic acid (Callebaut et al., 1990). The major anthocyanin of tradescantia was hypothesized to be cyanidin-3,7,3'-triglucoside with three molecules of ferulic acid, one molecule of caffeic acid and one terminal glucose (Shi et al., 1993). From the chromatograms presented, both tradescantia and ajuga contained more compounds that absorbed at $300 \mathrm{~nm}$, suggesting that intermolecular copigmentation may influence the stability of those extracts.

The stabilities of extracts exposed to light (Fig. 4) showed anthocyanins from tradescantia had greater stability compared to the others. The degradation pattern for tradescantia approached linearity, while in the other extracts an exponential degradation was apparent.

Peak areas for individual peaks separated by HPLC were used to follow degradation of individual anthocyanins over time (Table 1). After 15 days all pigments monitored from the four extracts with exception of tradescantia showed $\approx 90 \%$ degradation or higher. This shows that of all extracts, only tradescantia pigments displayed any reasonable stability to light under these conditions.

Tradescantia extract, because of its unusual stability, was further analyzed for known copigments such as rutin, chlorogenic acid and caffeic acid. Such copigments could aid in intermolecular stabilization. Copigments were analyzed by HPLC using the same elution conditions for tradescantia anthocyanins. Commercial standards were used to estimate concentrations of copigments found in the tradescantia extract and the ratio of copigments:pigment concentrations were summarized (Table 2). Davies and Mazza (1993) reported that $\mathrm{pH}$, copigment, pigment structure, and concentrations of the copigment and pigment have a strong influence on stability. The ratios of copigment:pigment concentration were low, which would indicate that intermole- 


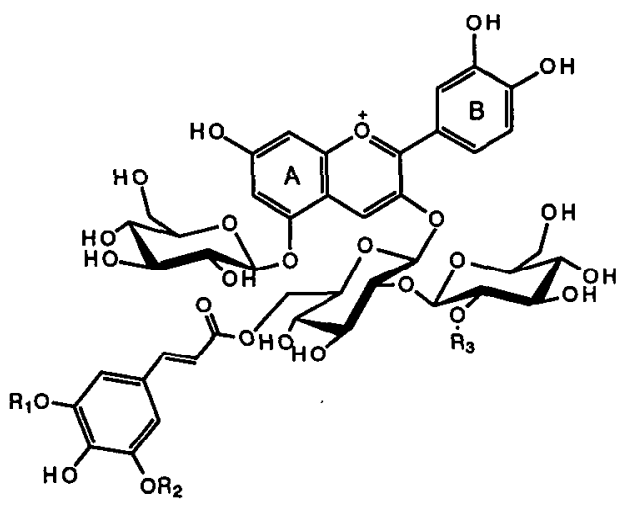

\begin{tabular}{lcccc}
\hline Peak & $\mathrm{R}_{1}$ & $\mathrm{R}_{2}$ & $\mathrm{R}_{3}$ & FABMS (m/z) \\
\hline 1 & $\mathrm{OCH}_{3}$ & $\mathrm{OCH}_{3}$ & $\mathrm{H}$ & 979.4 \\
2 & $\mathrm{OCH}_{3}$ & $\mathrm{H}$ & $\mathrm{H}$ & 949.2 \\
3 & $\mathrm{H}$ & $\mathrm{H}$ & $\mathrm{H}$ & 919.1 \\
4 & $\mathrm{OCH}_{3}$ & $\mathrm{OCH}_{3}$ & sinapyl & 1185.2 \\
5 & $\mathrm{OCH}_{3}$ & $\mathrm{H}$ & sinapyl & 1155.3 \\
6 & $\mathrm{H}$ & $\mathrm{H}$ & sinapyl & 1125.4 \\
\hline
\end{tabular}

Fig. 3-MS data of peaks identified in red cabbage extract.

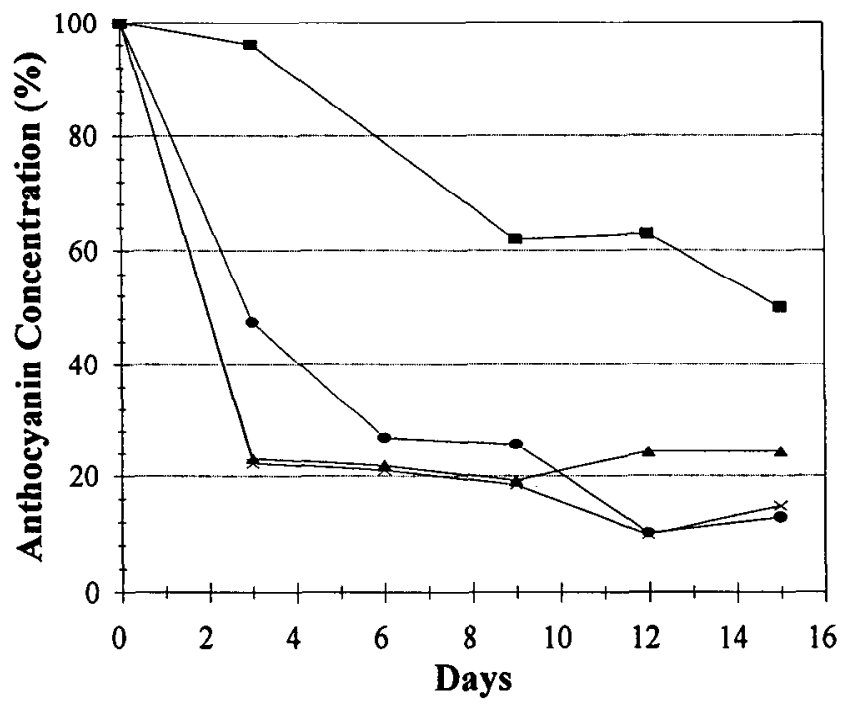

Fig. 4-Degradation of anthocyanin extracts over 15 days measured by spectroscopy. $\longrightarrow$ Concord grape; $X \longrightarrow$ ajuga; red cabbage; $\longrightarrow$ tradescantia.

cular copigmentation did not aid in stability of tradescantia extract. Chlorogenic based complexes fit a $1: 1$ (copigment: pigment) stochiometric ratio while that of caffeic acid is closer to a 0.5:1 ratio (Davies and Mazza, 1993). Also, copigmentation of a ring $B$ substituted anthocyanin has not been reported. A group in the B ring hydroxyl would make intermolecular copigmentation difficult due to steric hindrances. The increased stability of tradescantia anthocyanins was likely due to intramolecular copigmentation as suggested by the explanation of stability for Zebrina anthocyanin, a plant related to tradescantia (Brouillard, 1981).

The structure of anthocyanins from tradescantia are reported by Shi et al. (1992) to have B ring substitution, which aids in stability by preventing hydration and fading. Anthocyanins in both Concord grape and red cabbage are well characterized and no $B$ ring substitution has been reported. The structures of ajuga anthocyanins have not been completely elucidated, therefore spectral measurements were made to determine if that extract contained any $\mathbf{B}$ ring substituted anthocyanins. Spectral scans from 400 to $700 \mathrm{~nm}$ at $\mathrm{pH} 1,4$ and 7 were measured for tra-
Table 1-Degradation of individual anthocyanins from various extracts after being exposed to $250 \mu$ mole $\mathrm{sec}^{-1} \mathrm{~m}^{-2}$ light; $\mathrm{T}=30 \pm 1^{\circ} \mathrm{C}$

\begin{tabular}{|c|c|c|}
\hline Source & Anthocyanin ${ }^{a}$ & $\begin{array}{c}\text { \% Degradationb } \\
\text { after } \\
15 \text { Days }\end{array}$ \\
\hline Concord grape & $\begin{array}{c}\text { Cy Glc } \\
\text { Dp Glc } \\
\text { Dp Glc } p \text {-Cou }\end{array}$ & $\begin{array}{l}97 \\
94 \\
95\end{array}$ \\
\hline Red cabbage & $\begin{array}{c}\text { Cy (3)Gle } p \text {-Cou } \\
\text { Cy (3)Glc Fer } \\
\text { Cy (3)Glc Sin } \\
\text { Cy (3)Glc } p \text {-Cou Sin } \\
\text { Cy (3)Glc Fer Sin } \\
\text { Cy (3)Glc (2)Sin }\end{array}$ & $\begin{array}{l}99 \\
99 \\
99 \\
98 \\
98 \\
99\end{array}$ \\
\hline Ajuga & $\begin{array}{l}\text { Major peak } \\
\text { Minor peak }\end{array}$ & $\begin{array}{l}89 \\
86\end{array}$ \\
\hline Tradescantia & $\begin{array}{l}\text { Major peak } \\
\text { Minor peak }\end{array}$ & $\begin{array}{l}57 \\
36\end{array}$ \\
\hline
\end{tabular}

a Degradation determined by HPLC (\% area).

b $\mathrm{Cy}=$ cyanidin, $\mathrm{Dp}=$ delphinidin, Glc $=$ glucose, $p$-Cou $=$ coumaric acid, Fer $=$ ferulic acid and $\operatorname{Sin}=$ sinapic acid.

Table 2-Ratio of copigments in tradescantia extract to total pigment concentration

\begin{tabular}{lc} 
& Ratio \\
\multicolumn{1}{c}{ Copigment } & (copigment:pigment) \\
\hline Chlorogenic acid & $1: 42$ \\
Caffeic acid & $1: 3$ \\
Rutin & $1: 3$ \\
\hline
\end{tabular}
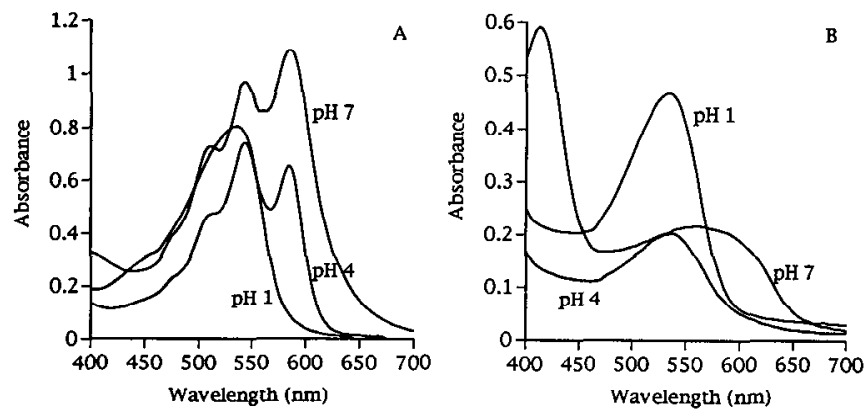

Fig. 5-Visible spectra of tradescantia extract (A) and ajuga extract (B) in buffer solutions at $\mathrm{pH} 1,4$ and 7 .

descantia and ajuga, (Fig. 5). Brouillard reported that for B ring substituted anthocyanins, lowered acidity instantaneously converted the cation to the quinonoidal base. Near pH 5.5 transformation to the quinonoidal base is complete, which is characterized by $\lambda$ max of 508,545 and $585 \mathrm{~nm}$ (Brouillard, 1981). It is clear that at pH 4 and above the tradescantia extract has these attributes for B ring substituted anthocyanins (Fig. 5). Ajuga does not show any additional absorbance with decrease in acidity, indicating that ajuga anthocyanins do not have $B$ ring substitution.

\section{CONCLUSIONS}

AMONG THE FOUR ANTHOCYANIN EXTRACTS studied, tradescantia anthocyanins showed greatest stability. The extraordinary stability of these anthocyanins could be attributed to their structure. Due to the high degree of acylation and substitution in the B ring of the chromophore, intramolecular copigmentation would decrease the hydration of these compounds to the colorless pseudobase which enhances the extract stability.

\section{REFERENCES}

Brouillard, R. 1981. Origin of the exceptional colour stability of the Zebrina anthocyanin. Phytochemistry 20: 143-145. 
Callebaut, A., Hendrickx, G., Voets, A.M., and Motte, J.C. 1990. Anthocyanins in cell cultures of Ajuga reptans. Phytochem. 29(7): 2153-2158.

Davies, A.J. and Mazza, G. 1992. Separation and characterization of anthocyanins of Monarda fistulosa by high-performance liquid chromatography. J. Agric. Food Chem. 40: 1341-1345.

Davies, A.J. and Mazza, G. 1993. Copigmentation of simple and acylated anthocyanins with colorless phenolic compounds. J. Agric. Food Chem. 41(5): 716-720.

Francis, F.J. 1992. A new group of food colorants. Trends in Food Sci. \& Technol. 3(2): 27-30.

Jurd, L. and Asen, S. 1966. The formation of metal and "co-pigment" complexes of cyanidin 3-glucoside. Phytochemistry 5: 1263-1271.

Labell, F. 1990. Technology diversifies use of natural colors. Food Processing 51(4): 69

Lea, A.G.H. 1988. HPLC of natural pigments in foodstuffs, In HPLC in Food Analysis, R. Macrae (Ed.), Academic Press Inc., San Diego, CA.

Mazza, G. and Miniati, E. 1993. Anthocyanins in Fruits, Vegetables, and Grains. CRC Press, Inc. Boca Raton, FL.
Shi, Z., Daun, H., and Francis, F.J 1993. Major anthocyanin from Tradescantia pallida: Identification by LSI-MS and chemical analyses. J. Food Sci. 58(4): 1-2.

Shi, $Z$. and Francis, F.J. 1992. Anthocyanins of Tradescantia pallida: poten tial food colorants. J. Food Sci. 57(3): 761-765.

Shi, Z., Lin, M. and Francis, F.J. 1992. Stability of anthocyanins from Tradescantia pallida. J. Food Sci. 57(3): 758-760.

Teh, L.S. and Francis, F.J. 1988. Stability of anthocyanins from Zebrina pendula and Ipomoea tricolor in a model beverage. J. Food Sci. 53: 1580 Wrolstad, R.E. 1976. Color and Pigment Analyses in Fruit Products. Agricultural Experiment Station, Oregon State Univ.

cultural Experiment Station, Oregon State Univ.
Ms received 3/30/94; revised 6/16/94; accepted 7/8/94.

This work was supported by a grant trom Ag 92-37500-8146 USDAPVAP and CHE-9250021 NSF/MRI. We are thankful to San-Fy chemical industries and Stresling wineries for the samples used in this studs. 